\title{
Les réformes éducatives en cours au Sri Lanka
}

Anne-Marie Bardi

\section{OpenEdition}

\section{Journals}

Édition électronique

URL : http://journals.openedition.org/ries/3977

DOI : 10.4000/ries.3977

ISSN : 2261-4265

\section{Éditeur}

Centre international d'études pédagogiques

\section{Édition imprimée}

Date de publication : 1 septembre 2014

Pagination : 14-20

ISBN : 978-2-85420-604-3

ISSN : 1254-4590

\section{Référence électronique}

Anne-Marie Bardi, «Les réformes éducatives en cours au Sri Lanka », Revue internationale d'éducation de Sèvres [En ligne], 66 | septembre 2014, mis en ligne le 01 septembre 2016, consulté le 22 juin 2020 URL : http://journals.openedition.org/ries/3977 ; DOI : https://doi.org/10.4000/ries.3977

Ce document a été généré automatiquement le 22 juin 2020.

(c) Tous droits réservés 


\title{
Les réformes éducatives en cours au Sri Lanka
}

\author{
Anne-Marie Bardi
}

1 En moins de soixante-dix ans d'indépendance, le Sri Lanka a connu de fortes évolutions politiques, économiques, sociales et éducatives. Le «Cadre pour le développement du secteur éducatif» (Education sector development framework) pour la période 2012-2016 porte le sous-titre évocateur «Transformer l'éducation scolaire pour fonder le projet de hub de la connaissance» (Transforming School Education as the Foundation of a Knowledge Hub Project), qui inscrit pleinement l'éducation dans le projet de développement économique du pays. Pour saisir l'aspect stratégique de ces nouvelles orientations, il n'est pas inutile de disposer de quelques repères historiques et d'une brève description de la situation qui, au début du xxI ${ }^{\mathrm{e}}$ siècle, a conduit à adopter ce plan.

\section{L'héritage de la période coloniale}

Le Sri Lanka, île située à une trentaine de kilomètres au sud-est de l'Inde sur une route maritime fréquentée, a connu une colonisation portugaise, hollandaise puis britannique de près de quatre cents ans, depuis le début $d u \mathrm{XvI}^{\mathrm{e}}$ siècle jusqu'à son indépendance en 1948. Dès le début du XIXe siècle, les Britanniques y ont développé une importante industrie de culture et d'exportation de matières premières, essentiellement le thé et l'hévéa, et mis en place une forte administration locale, nombreuse et centralisée. Tandis que l'enseignement public de base était effectué, selon les écoles, dans l'une ou l'autre des deux langues nationales (cinghalais ou tamoul), l'éducation de la classe moyenne et des futures élites relevait d'établissements tenus par des congrégations religieuses chrétiennes, établissements aidés par l'État mais payants et où tous les cours étaient donnés en anglais. Cette éducation était la voie unique d'accès à tous les postes administratifs, très convoités car assurant la sécurité de l'emploi et la promotion sociale, ceux-ci exigeant une pratique courante de l'anglais. Ce monopole chrétien n'a pas manqué de créer des tensions entre communautés et, au 
début $\mathrm{du} \mathrm{xx}^{\mathrm{e}}$ siècle, des écoles fondées sur d'autres identités ethno-religieuses et soutenues financièrement par l'État apparurent: en 1920, elles étaient à $25 \%$ bouddhistes, $10 \%$ hindouistes et $5 \%$ musulmanes, les autres chrétiennes. L'enseignement y était toujours donné en anglais. Dans les écoles primaires publiques de base, où l'enseignement était donné soit en cinghalais soit en tamoul, des tentatives eurent lieu dans les années 1930 pour introduire un enseignement de l'autre langue (sous la forme d'une seconde langue) afin de permettre de meilleurs échanges entre les populations. Ce fut un échec, chaque communauté refusant d'apprendre la langue de l'autre, et donc un frein à la mobilité géographique. Le renforcement des divisions ethno-religieuses, sociales et linguistiques dû à l'éducation est donc au Sri Lanka un phénomène ancien et marquant.

\section{Le Sri Lanka aujourd'hui}

C'est un pays de 21 millions d'habitants (projection à 23 millions en 2050), dont la population est à $85 \%$ rurale et dont un quart (26\%) est âgé de 14 ans et moins. Le PIB par habitant est inférieur à celui Thaïlande et de la Chine mais supérieur à celui de l'Indonésie, de l'Inde, du Vietnam, et plus de deux fois supérieur à celui du Laos, du Pakistan ou du Cambodge. L'économie repose à $57 \%$ sur les services et à $32 \%$ sur l'industrie. Le PIB croit de 6,4\% par an. L'indice de développement humain calculé par le PNUD situe le Sri Lanka en $92^{\mathrm{e}}$ place, devant la Chine, la Thaïlande, l'Indonésie, le Vietnam et l'Inde, notamment.

4 Après l'indépendance, la création d'un État centralisé n'a pas aboli les oppositions entre cinghalais ( $74 \%$ de la population aujourd'hui, essentiellement dans le sud et le centre du pays) et tamouls (7,5\%, dans le nord et l'est) et a parfois, bien au contraire, exacerbé les tensions entre populations majoritaires et minoritaires, jusqu'à conduire à un terrible conflit ethnique de près de trente ans qui n'a pris fin officiellement qu'en 2009 et laisse encore de profondes traces.

5 Aujourd'hui, le cinghalais est la langue officielle nationale; le tamoul est aussi langue nationale et l'anglais demeure la "langue de liaison». L'anglais est utilisé dans les instances gouvernementales mais n'est maîtrisé à bon niveau que par $10 \%$ de la population.

\section{Les principes et les finalités de l'enseignement}

La constitution du Sri Lanka (1978) reconnait le droit universel de tous les citoyens à l'éducation à tous les niveaux. Les grands objectifs de la politique éducative sont :

- développer et comprendre l'héritage culturel et religieux en appréciant l'apport des différentes ethnies du pays ;

- développer des attitudes menant à une cohésion sociale entre les différentes communautés ;

- promouvoir le développement moral, spirituel et physique et inculquer un sens du devoir envers la patrie ;

- développer et promouvoir un système pour l'acquisition des savoirs techniques et des compétences long de la vie ;

- promouvoir la démocratisation de l'éducation. 


\section{L'administration de l'éducation}

7 Le ministère central de l'éducation supervise :

- le Département des examens et des tests nationaux, chargé des procédures d'examens ;

- l'Institut national d'éducation (National Institute of Education - NIE), responsable de la formation des enseignants et des cadres de l'éducation, du développement des curricula et de la recherche en éducation;

- la Commission nationale de l'éducation, mise en place en 1991, qui conseille le Président en matière de politique éducative, afin d'assurer une continuité dans la politique éducative et une réponse adéquate aux besoins changeants du pays.

8 Depuis 1987, le système éducatif a une gestion décentralisée. Le ministère de l'éducation est responsable des politiques et programmes nationaux, de la gestion des écoles dites nationales, de la formation des enseignants et de la qualité des standards dans les écoles. La mise en œuvre des politiques éducatives relève de plus en plus de chacune des neuf provinces, dotées d'un ministère de l'éducation provincial et chargées de la gestion des écoles dites provinciales. Des zones éducatives sont définies et placées sous l'autorité d'un bureau de zone (Zonal Education Office) qui supervise et administre les cent à cent cinquante écoles de sa zone.

Le ministère de l'enseignement supérieur a été créé en 2011.

Le ministère du développement des compétences, de l'enseignement technique et professionnel met en œuvre les stratégies en collaboration avec les différents partenaires de l'éducation et de la formation professionnelle.

La National Apprentice and Industrial Training Authority dirige des programmes de formation nationaux pour des petites, moyennes ou grandes entreprises.

Le gouvernement consacre moins de $2 \%$ du PIB à l'éducation, ce qui représente seulement $9 \%$ des dépenses des administrations publiques; ce sont les plus faibles pourcentages de tous les pays de la zone. Il y a environ 1,7 million d'élèves scolarisés dans le primaire (dont 4,5\% dans le privé) et 2,6 millions dans le secondaire (dont 7,3\% dans le privé).

\section{L'organisation du système éducatif}

On distingue trois types d'écoles financées par l'État:

- les écoles nationales, avec un contrôle et un financement directs du ministère de l'éducation : pour la plupart elles sont issues du système colonial, ce sont des institutions établies et fréquentées par les élites, extrêmement recherchées ;

- les écoles provinciales, qui représentent la grande majorité des écoles : elles sont financées et gérées par les gouvernements locaux et souffrent d'un manque d'équipements et d'enseignants ;

- les Pirivenas, écoles monastiques bouddhistes, des centres d'enseignement secondaire et supérieur traditionnels très anciens; 561 Pirivenas sont financées par le ministère de l'éducation.

13 La prise en charge des enfants de 3 à 5 ans relève d'initiatives locales, d'organismes religieux, d'associations ou d'institutions privées. On estime que près de $90 \%$ des enfants fréquentent ces établissements mais de manière souvent épisodique. 

(classes 1 à 5) qui s'achève par un examen national et dont les résultats peuvent permettre d'obtenir une bourse pour aller dans de meilleures écoles, puis du secondaire inférieur (classes 6 à 9). En 2005 on évaluait à $92 \%$ la proportion de jeunes scolarisés dans l'enseignement obligatoire public (94\% au primaire, $87 \%$ au secondaire) avec une représentation à peu près égale des garçons et des filles. Le secondaire supérieur (classes 10 et 11) s'achève par le General Certificate of Education Ordinary level (GCEO/L), nécessaire pour entrer au niveau «collegiate» (classes 12 et 13) et passer le General Certificate of Education Advanced Level (GCEA/L), qui, lui-même, détermine l'entrée à l'université. La $11^{\mathrm{e}}$ année, les élèves peuvent être dirigés vers l'enseignement technique et professionnel. offrent toutes les séries (sciences, économie et arts) jusqu'au GCEA/L (fin du college classe 13) ; ceux de type $1 \mathrm{C}$ font de même mais seulement pour les filières économie et arts; ceux de type 2 ne vont que jusqu'au GCEO/L (fin du secondaire supérieur classe 11) et ceux de type 3 ne vont que jusqu'à la $5^{\mathrm{e}}$ année du primaire.

L'éducation publique est gratuite. Les manuels sont gratuits jusqu'à la fin du college. Il n'y a pas de cantine ni de prise en charge des repas.

Les établissements publics sont majoritaires, mais il existe des écoles privées, qui suivent le curriculum national dans les langues d'enseignement nationales (cinghalais, tamoul ou anglais). Certaines écoles privées sont avec frais de scolarité (sans subvention de l'État) et d'autres sans frais de scolarité car subventionnées.

L'attente éducative des familles et des jeunes est extrêmement forte, ce qui conduit à un développement rapide et très important des leçons particulières et des officines de soutien scolaire. Tous les milieux sociaux sont concernés ou presque, et la répétition après la classe est très fréquente, surtout les années s'achevant par un examen national (GCE/O et GCE/A). Selon les ressources des familles, le tutorat est effectué individuellement, par petits groupes, parfois en très grands groupes par des animateurs plus ou moins compétents, par les professeurs parfois. Le gouvernement a même dû interdire aux enseignants d'État de donner des leçons à titre privé durant les horaires scolaires.

\section{Le corps enseignant}

19 Les enseignants pour les années 1 à 11 sont recrutés avec un diplôme obtenu après deux années d'études au college of education et une année de stage en établissement. Les enseignants des niveaux 12 et 13 sont diplômés de l'université. Dans les années 1990, la massification $\mathrm{du}$ système éducatif a entraîné le recrutement de près de 55000 enseignants non formés, diplômés de l'université ou non. Des programmes de formation à distance et de formation continue ont été mis en place à travers un vaste réseau composé du NIE, de départements universitaires, des colleges of education et de centre provinciaux et locaux. Les réformes de curriculum ont également été accompagnées de formations tant pédagogiques (modalités d'évaluation par exemple) que disciplinaires (anglais, informatique, etc.).

Sur un corps enseignant de 216000 personnes, 183000 exercent dans des établissements sous autorité provinciale (21000 dans le primaire, 67000 dans les 
établissements de type 2, 69000 dans les établissements de type 1C et 58000 dans les établissements de type $1 \mathrm{~A}$ et $1 \mathrm{~B}$; trois quart d'entre eux enseignent en cinghalais, $24 \%$ en tamoul et $1 \%$ en anglais.

\section{L'enseignement supérieur}

21 L'enseignement supérieur est gratuit mais extrêmement compétitif : moins de $16 \%$ de ceux qui obtiennent le GCEA/L sont admis dans les universités d'État. Seuls les meilleurs de chaque district vont à l'université et seule la moitié d'entre eux obtiennent leur diplôme du supérieur. Il existe quinze universités d'État, seules habilitées à délivrer des diplômes nationaux.

Les évolutions du système éducatif n'ont pas été aussi rapides que celles du secteur économique. Celui-ci requiert de plus en plus de jeunes anglophones formés au management et aux techniques de production et de gestion modernes. Cela explique le grand nombre de systèmes parallèles de formation et de délivrance de diplômes étrangers installés au Sri Lanka. Le Royaume-Uni tient une place majeure en ce domaine. Le British Council y joue un rôle important, délivrant cours et qualifications de langue mais abritant, de plus, des cours et des passations d'examens britanniques (GCE/O et GCE/A) et des diplômes d'université, de Londres et de Cambridge notamment. Ainsi, moyennant finances, il est possible de poursuivre des études supérieures et d'obtenir des diplômes internationaux appréciés des employeurs pour travailler dans des entreprises multinationales, sans aller suivre de cours à l'étranger.

\section{Les contenus d'enseignement}

En 1997, une révision du curriculum de l'enseignement primaire a mis l'accent sur les compétences de communication, l'étude de l'environnement social et naturel, l'éthique et la religion, et la composante "apprendre à apprendre». Les méthodes d'enseignement ont privilégié l'activité des élèves plus que l'écoute du maitre. Ces réformes ont été mises en place progressivement jusqu'en 2003.

Les matières enseignées au primaire et les horaires hebdomadaires sont: la langue (cinghalais ou tamoul) : $5 \mathrm{~h}$; l'anglais (à partir de la classe 3): $3 \mathrm{~h}$, l'autre langue nationale (à partir de la classe 3 ) $: 1 \mathrm{~h}$; les mathématiques $3 \mathrm{~h} 30$ puis $5 \mathrm{~h}$; les activités liées à l'environnement (sciences sociales, santé, physique, musique, danse, dessin) $6 \mathrm{~h}$ puis $7 \mathrm{~h} 30$; la religion $1 \mathrm{~h} 15$ puis $2 \mathrm{~h}$. Si on ajoute les récréations, les assemblées du matin, les activités religieuses et sportives, la semaine de classe dure de $20 \mathrm{~h}$ (classes 1 et 2) à $29 \mathrm{~h}$. Les enseignants ont été encouragés à développer une évaluation par compétences au cours de l'année. Les résultats à l'examen de fin de primaire montrent une très grande disparité de résultats entre écoles rurales et urbaines, comme entre enfants de familles aisées ou à bas revenus.

Entre 2001 et 2007, les contenus des programmes du secondaire ont été revus, avec l'intention de renforcer une éducation à la paix et à l'harmonie nationale, aux principes démocratiques, aux droits de l'homme, à l'égalité des genres et la préservation de l'environnement. La volonté de préparer des jeunes ouverts sur le monde du travail a conduit à introduire une nouvelle matière, la technologie, et à privilégier des compétences telles que le travail en équipe, la communication, la résolution de 
problèmes ou l'esprit d'entreprise. Ceci s'est accompagné du développement de laboratoires de sciences, de ressources pour les langues vivantes et de l'usage des technologies de l'information et de la communication. De la classe 6 à la classe 11, la semaine d'enseignement compte 40 heures. En parallèle à la rénovation des contenus, les compétences attendues à chaque étape ont été précisées, avec deux niveaux (essentiel et souhaitable) et les enseignants ont été encouragés à intégrer l'évaluation dans leur processus d'enseignement.

\section{Le nouveau cadre pour le développement du secteur éducatif}

Après avoir expérimenté un programme de développement du secteur éducatif conforme aux recommandations et aux standards de la Banque mondiale entre 2006 et 2011, le gouvernement du Sri Lanka met en place un nouveau Cadre pour le développement du secteur éducatif, qui couvre la période 2012-2016 et s'intitule "Transformer l'éducation scolaire pour fonder le projet de hub de la connaissance ». Ce plan, assorti de ses règles de financement et toujours conforme aux recommandations de la Banque mondiale, vise à augmenter l'accès à l'enseignement primaire et secondaire, à en améliorer la qualité et à construire les fondations d'une économie et d'une société de la connaissance pour le pays. Il concerne les neuf régions et repose sur trois piliers :

\section{Pilier 1 : promouvoir l'égalité d'accès à l'éducation primaire et secondaire}

Tous les enfants âgés de 6 à 16 ans doivent pouvoir suivre une scolarité complète de onze années et aller dans un établissement secondaire qui propose toutes les filières (arts, sciences, management). Pour cela, dans chaque province sont identifiés les ensembles d'écoles primaires qui alimentent chaque école secondaire. Il y aura à terme une école primaire par village et une école secondaire à distance raisonnable. Des aides seront données pour l'achat des uniformes et des efforts seront faits en matière de santé scolaire et de nutrition, allant jusqu'à la fourniture de repas pour les enfants des familles les plus démunies. On veillera à disposer de sanitaires dédiés pour les jeunes filles. Des programmes spécifiques d'éducation spéciale ou non formelle seront mis en place pour les enfants qui en ont besoin, particulièrement ceux du nord et de l'est qui sortent de près de trente années de violences traumatisantes.

\section{Pilier 2 : améliorer la qualité de l'éducation}

Cela repose sur une amélioration de l'évaluation à tous niveaux : en continu dans les classes, au plan national et même au plan international. Un institut de recherche en éducation et évaluation a été créé à l'université de Colombo. Il doit travailler aux évaluations nationales mais aussi permettre au Sri Lanka de participer désormais aux évaluations internationales de type TIMSS et PISA, fournissant ainsi des informations précieuses aux décideurs afin de faire évoluer les curricula, la formation initiale et continue des enseignants, la production de ressources pédagogiques etc. Les 
évaluations locales permettront de plus aux autorités provinciales de mesurer la qualité des établissements de leur région.

Une attention particulière est donnée à la promotion de l'enseignement des sciences, des mathématiques, des technologies de l'information et de l'anglais. Le " programme d'éducation bilingue » incite à enseigner certaines matières en anglais au secondaire, dans le but de disposer de jeunes plus nombreux maitrisant cette langue. Les évaluations nationales porteront sur ces matières. Sur de telles bases, le Sri Lanka compte devenir un véritable carrefour dans une société de la connaissance (knowledge hub with knowledge based services). Le curriculum est aussi perçu comme le moyen de présenter une image positive de la pluralité ethnique et culturelle du pays, de provoquer des interactions entre élèves et de préparer ainsi la stabilité sociale et politique du pays.

\section{Pilier 3 : renforcer la gouvernance}

Désormais le management des écoles n'est plus uniquement confié aux chefs d'établissements, aux enseignants et à l'administration. Après une période expérimentale évaluée positivement, il a été décidé que se joignent désormais à eux les parents d'élèves, les anciens élèves et toutes les personnes de bonne volonté. L'école dispose d'une relative autonomie et peut lever des fonds localement; cela doit lui permettre de développer des actions extra-curriculaires visant le développement de nouvelles compétences (travail en équipe, communication, éthique, esprit d'entreprise, etc.). Dans un souci de transparence, les comptes sont rendus publics chaque année ainsi qu'un bilan présentant les objectifs atteints et les initiatives prises pour progresser.

31 Les compétences administratives et de gestion des chefs d'établissement - qui se voient confier de lourdes responsabilités dans cette nouvelle relative autonomie - et celles des responsables de zones et d'échelons provinciaux sont à développer et à renforcer.

L'évaluation et le pilotage de cette réforme sont un point important. Y sont associées tant les agences et universités d'Etat que des agences d'évaluation privées indépendantes. Le financement de la réforme repose sur des fonds du gouvernement du Sri Lanka et sur ceux de la Banque Mondiale. Leur attribution est régulée par les résultats des évaluations.

En moins de soixante-dix années d'indépendance, après des changements politiques et économiques radicaux, après avoir vu la fin de conflits armés meurtriers, le Sri Lanka promeut une réconciliation nationale, adopte un modèle économique libéral, s'inscrit dans la mondialisation et cherche à se développer en tant que carrefour reconnu dans l'économie de la connaissance. Les réformes éducatives en cours sont un des fondements de ce projet. Une lecture en creux montre que, si la scolarisation obligatoire est une réussite quantitative, les questions de qualité de l'enseignement, d'égalité d'accès aux diverses filières et d'ouverture de l'enseignement supérieur demeurent à résoudre. Les orientations actuelles révèlent une vraie ambition; la volonté d'évaluer, à tous niveaux, les effets de la réforme en cours devraient aider à sa réussite. 


\section{BIBLIOGRAPHIE}

LITTLE Angela W., HETTIGE Siri T. (2013): Globalisation employment and education in Sri Lanka: opportunity and division. Londres : Routledge.

UNESCO, International Bureau of Education (2011): World data on education. VII ed. 2010/11 : SriLanka, disponible sur : http://www.ibe.unesco.org/fileadmin/user_upload/Publications/WDE/ 2010/pdf-versions/Sri_Lanka.pdf

UNESCO Bangkok (2013): Financing of secondary education in the Asia-Pacific region: synthesis paper, UNESCO Bangkok Office, disponible sur: http://unesdoc.unesco.org/images/

0022/002255/225507e.pdf

\section{INDEX}

Mots-clés : politique éducative, réforme, système éducatif

Index géographique : Sri Lanka

Keywords : educational system, educational policy, reform

Palabras claves : política educacional, reforma, sistema educativo

\section{AUTEUR}

\section{ANNE-MARIE BARDI}

Anne-Marie Bardi est inspectrice générale honoraire de l'éducation nationale (France). Ancienne élève de l'École normale supérieure de Fontenay-aux-Roses, agrégée de mathématiques, elle a été professeur puis inspectrice d'académie-inspectrice pédagogique régionale de mathématiques. Elle s'est notamment beaucoup impliquée dans le développement de l'usage des technologies de l'information et de la communication dans l'enseignement en France et à l'étranger. 